ORIGINAL RESEARCH

H.P. Burmeister

P.A. Baltzer

M. Dietzel

I. Krumbein

T. Bitter

A. Schrott-Fischer

O. Guntinas-Lichius

W.A. Kaiser

\title{
Identification of the Nervus Intermedius Using 3T MR Imaging
}

BACKGROUND AND PURPOSE: Improved MR imaging at higher field strengths enables more detailed imaging of cranial nerves. The aim of this study was to assess the identifiability of the $\mathrm{NI}$ in the CPA and IAC by using high-resolution 3T MR imaging.

MATERIALS AND METHODS: Twenty-seven healthy volunteers (13 men and 14 women; mean age, 33 years) underwent $3 T$ MR imaging of the CPA. The section thicknesses of the CISS sequence was 0.4 $\mathrm{mm}$ (TR, $12.18 \mathrm{~ms}$; TE, $6.09 \mathrm{~ms}$ ) using a 12-channel head coil. Evaluation was performed by using MPR mode. Image quality and identifiability of the NI were rated independently by 2 observers according to predefined criteria on an ordinal scale. Interobserver agreement was assessed by $\kappa$ statistics.

RESULTS: Fifty-four NIs were evaluated. Both observers were able to identify the $\mathrm{NI}$ in nearly $60 \%$ of cases. It was possible to indentify at least $1 \mathrm{NI}$ in $70 \%$ of all volunteers in the CPA and/or IAC. Image quality ratings showed a substantial agreement $(\kappa=0.65)$ and identifiability ratings an almost perfect $(\kappa=0.83)$ agreement.

concLusions: Careful evaluation of all nervous and vascular structures in the CPA and IAC at high-resolution 3T MR imaging allows reliable depiction of the NI.

ABBREVIATIONS: $\mathrm{A}=$ anterior; $\mathrm{CISS}=$ constructive interference in steady state; $\mathrm{CN}=$ nervus cochlearis; CPA = cerebellopontine angle; $\mathrm{FN}=$ nervus facialis; I = inferior; IAC = internal auditory canal; $\mathrm{MPR}=$ multiplanar reconstruction; $\mathrm{NI}=$ nervus intermedius; $\mathrm{P}=$ posterior; $\mathrm{S}=$ superior; $\mathrm{SNR}=$ signal intensity-to-noise ratio; $\mathrm{VI}=$ nervus vestibularis inferior; $\mathrm{VS}=$ nervus vestibularis superior

$T$ he NI contains sensory and parasympathetic fibers that innervate the parotid, submandibular, submental, and minor palatine and pharyngeal salivary glands as well as the lacrimal glands. The NI is also responsible for the sensation of taste in the anterior two-thirds of the tongue. The NI originates at the brain stem between the facial nerve and the vestibulocochlear nerve in the lateral medullopontine sulcus. ${ }^{1}$ In its further course, the NI accompanies the facial nerve or the vestibulocochlear nerve. In the latter case, it crosses over to the facial nerve at the level of the internal auditory meatus. Anatomic studies ${ }^{2}$ revealed multiple variations of the NI in the CPA and IAC, both in its origin and course.

Until now, depiction of the NI by imaging was not possible by using either CT or MR imaging at $1.5 \mathrm{~T} .^{3}$ One major advantage of high-field MR imaging (ie, at 3T) is an increased SNR. This higher SNR results in better spatial resolution. ${ }^{4}$ Data on a possible improvement of imaging of the CPA at 3T, in particular the NI, ${ }^{5}$ are limited. Consequently, this study investigated the hypothesis that imaging the NI in the CPA and IAC is possible by using $3 \mathrm{~T}$ MR imaging.

Received May 5, 2010; accepted after revision August 20.

From the Institute of Diagnostic and Interventional Radiology (H.P.B., P.A.B., M.D., I.K., W.A.K.) and Department of Otorhinolaryngology (T.B., 0.G.-L.), University Hospital, Friedrich Schiller University, Jena, Germany; and Department of Otorhinolaryngology (A.S.-F.), University of Innsbruck, Innsbruck, Austria.

Please address correspondence to Hartmut Peter Burmeister, MD, Institute of Diagnostic and Interventional Radiology, University Hospital, Friedrich Schiller University Jena, Philosophenweg 3, D-07740 Jena, Germany; e-mail: hartmut.burmeister@med.uni-jena.de

एक्ष

Indicates article with supplemental on-line figures.

DOI 10.3174/ajnr.A2338

\section{Materials and Methods}

\section{Participant Recruitment and Sampling}

In this prospective observational study, we examined a consecutive series of 27 healthy volunteers. Inclusion criteria were as follows: at least 18 years of age; no symptoms of common cold, allergic rhinitis, or infections relating to the temporal bone or respiratory tract; no medication influencing fluid balance or blood pressure; and a subjective feeling of well-being. Volunteers not eligible for this investigation were those with known contraindications against MR imaging or any known previous surgery or disease of the CPA or temporal bone. Institutional review board approval was obtained, and all volunteers gave written informed consent for the examination.

\section{Imaging Procedures}

All volunteers underwent a 3T MR imaging examination (Magnetom Tim Trio; Siemens, Erlangen, Germany) of the temporal bone in our institution. The voxel size of the axial 3D-CISS Fourier transformation sequence ${ }^{6}$ was $0.4 \times 0.4 \times 0.4 \mathrm{~mm}(\mathrm{TR}, 12.18 \mathrm{~ms}$; TE, $6.09 \mathrm{~ms}$; matrix, $512 \times 512$; FOV, $210 \mathrm{~mm}$; flip angle, $50^{\circ}$; bandwidth, 130 $\mathrm{Hz} /$ pixel; averages, 1; time of acquisition, 8 minutes and 8 seconds) using a dedicated 12 -channel head coil provided by the manufacturer. The volunteers were examined in a supine position; section orientation was parallel to the hard palate.

\section{Data Analysis}

Acquired images were transferred to a workstation (Syngo software, MR B15 Numaris/4; Siemens) for analysis.

On the basis of known anatomic characteristics of the encephaloperipheral nervous system and its vascularization in the CPA and $\mathrm{IAC}^{7}$ structures in the CPA and IAC were characterized as nerves (ie, nervus facialis, nervus intermedius, nervus vestibularis superior, nervus vestibularis inferior, and nervus cochlearis) or vessels (ie, arteria 
cerebelli inferior anterior, arteria labyrinthi, and vena labyrinthi). For identification of the NI, MPRs with each section orientation indicted on the other reconstruction planes as color-coded cross hairs were used (On-Line Fig 1). This approach allows exact correlation of anatomic structures in different planes.

Two radiologists, the first (H.P.B.) specializing in temporal bone imaging (observer one, 7 years' experience), and the other (P.A.B.) experienced in general MR imaging (observer two, 3 years' experience) evaluated all images. Both independently rated the image quality and identifiability of the NI in the CPA and IAC. Image quality was categorized as follows: $0=$ insufficient, $1=$ adequate, $2=$ excellent.

Criteria for insufficient rating of image quality were pronounced occurrence of movement, pulsation, off-resonance, or banding artifacts preventing reliable evaluation due to duplication or disappearance of anatomic structures.

The image quality was rated as "adequate" if the following criteria were present: mild occurrence of artifacts, no duplications or disappearances, and continuous delineation of nervous or vascular structures. The image quality was rated as "excellent" if no or insignificant artifacts were observed, allowing excellent visualization of nervous or vascular structures.

Identifiability of the NI was also categorized according to an ordinal scale: $0=$ none, $1=$ adequate, $2=$ precise. A negative identifiability rating was assigned if image quality was insufficient or anatomic structures did not meet the criteria listed below.

The identifiability was rated as adequate by applying the following criteria: linear anatomic structure either in the CPA or IAC, minimal length one-third of the CPA or IAC, visually estimated diameter $30 \%-70 \%$ of the diameter of the facial nerve, ${ }^{8}$ either parallel alignment to the nervus facialis and vestibulocochlearis or crossing over from the vestibulocochlear to the facial nerve (not vice versa), localization between the facial and vestibulocochlear nerve in the upper part of the IAC, no vessel contact capable of being misinterpreted in 2 of 3 planes by using MPR, and no vessel-like tortuosity or loops.

The identifiability was rated as "precise" if the following criteria were present: linear anatomic structure in the CPA and/or IAC, length exceeding one-third of the CPA or IAC, visually estimated diameter $30 \%-70 \%$ of the diameter of the facial nerve, either parallel alignment to the nervus facialis and vestibulocochlearis or crossing over from the vestibulocochlear to the facial nerve (not vice versa), localization between the facial and vestibulocochlear nerve in the upper part of the IAC, no vessel contact capable of being misinterpreted in 2 of 3 planes by using MPR modus, and no vessel-like tortuosity or loops.

Additional criteria corroborating the classification of a structure as NI were a delimitation of the origin in the medullopontine sulcus and converging with the nervus facialis right before entering the fallopian canal.

\section{Statistical Analysis}

All data analysis was performed by using the Statistical Package for the Social Sciences, Version 17.0 for Windows (SPSS, Chicago, Illinois) by calculation of frequency tables and cross tables. Interobserver agreement of image quality and identifiability ratings were assessed by $\kappa$ statistics. Results were benchmarked according to the classification of Landis and Koch for measurements of observer agreement for categoric data.

\section{Results}

Fifty-four NIs of 27 healthy volunteers were evaluated (13 men and 14 women) in this prospective study in February 2009. All

\begin{tabular}{llccrr}
\hline \multicolumn{5}{l}{ Table 1: Cross-tabulations of image-quality ratings ${ }^{\mathrm{a}}$} \\
\cline { 2 - 4 } & \multicolumn{3}{c}{ Observer B } & & \\
\cline { 2 - 5 } Image Quality & 0 & 1 & 2 & Total & $\%$ A \\
\hline Observer A & 1 & 0 & 0 & 1 & 1.9 \\
0 & 2 & 12 & 2 & 16 & 29.6 \\
1 & 0 & 5 & 32 & 37 & 68.5 \\
2 & 3 & 17 & 34 & 54 & 100 \\
Total & 5.5 & 31.5 & 63.0 & 100 & \\
$\%$ B &
\end{tabular}

a 0 indicates insufficient; 1 , adequate; 2 , excellent.

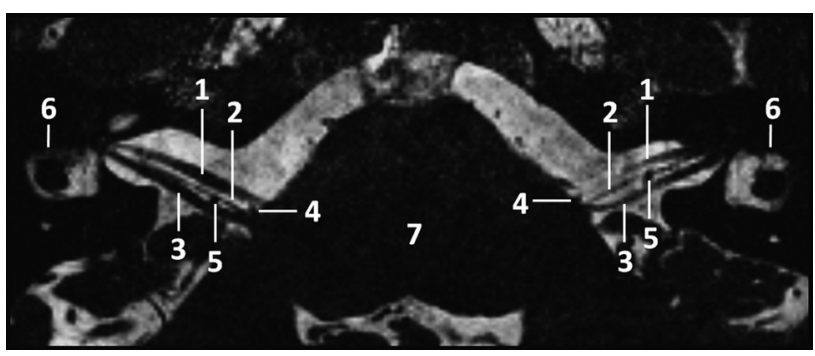

Fig 1. Para-axial CISS sequence image (TR, $12.18 \mathrm{~ms}$; TE, $6.09 \mathrm{~ms}$; flip angle, $50^{\circ}$ ) of the CPA at $3 \mathrm{~T}$ depicting the infrequent finding of an approximate symmetric course of the $\mathrm{Nl}$ : 1) nervus facialis; 2) nervus intermedius; 3) nervus vestibulocochlearis; 4) loop of the anterior inferior cerebellar artery between the origin of 1 and 2; 5) loop of the anterior inferior cerebellar artery dorsal of the nervus intermedius; 6 ) lateral semicircular canal; and 7) brain stem.

volunteers met the inclusion and exclusion criteria. The volunteers' ages were between 22 and 76 years (mean, 33 years). No sex-specific differences regarding image quality or identifiability of the NI were identified during the evaluation process.

\section{Image Quality}

Only $1.9 \%-5.5 \%$ artifacts lead to an insufficient image quality. An adequate image quality ranged between $29.6 \%$ and $31.5 \%$; and excellent image quality, between $62.9 \%$ and $68.5 \%$ of the 54 NIs (Table 1 ). According to $\kappa$ statistics, interobserver agreement of the image quality rating was substantial $(\kappa=0.65)$.

\section{Identification of the NI}

In 8 volunteers, the NI could be identified neither on the right nor on the left side (29.6\%). In $25.9 \%$ (7 volunteers), a unilateral identification and in $44.5 \%$ (12 volunteers) a bilateral identification of the NI were possible. Regarding the categorization of identifiability ratings, the NI was identified adequately in $22.2 \%$ and identified precisely in $35.2 \%$. In cases of bilateral findings, in $22.3 \%$ (6 volunteers), the raters were in agreement regarding the categorization of the identifiability rating. However, in none of the cases did the course of the nerves show an exact symmetry when comparing both sides. In only 1 case, was an approximately symmetric course found (Fig 1).

At least 1 NI could be identified in $70.4 \%$ of all volunteers, and the case-related overall identifiability of the nerve was $57.4 \%$ (Table 2). In only $3.7 \%$ of the cases were there differences in the assessment regarding the identifiability of the NI and in $7.4 \%$, regarding the quality of identifiability. Interobserver agreement of identifiability $(\kappa=0.83)$ and overall identifiability $(\kappa=0.92)$ of the NI in the CPA and the IAC were almost perfect. 


\begin{tabular}{lcccrc}
\hline \multicolumn{5}{l}{ Table 2: Cross-tabulations of identifiability ratings ${ }^{\mathrm{a}}$} \\
\hline & \multicolumn{3}{c}{ Observer B } & & \\
\cline { 2 - 4 } Identifiability & 0 & 1 & 2 & Total & $\%$ A \\
\hline Observer A & 22 & 1 & 0 & 23 & 42.6 \\
0 & 1 & 9 & 2 & 12 & 22.2 \\
1 & 0 & 2 & 17 & 19 & 35.2 \\
2 & 23 & 12 & 19 & 54 & 100 \\
Total & 42.6 & 22.2 & 35.2 & 100 & \\
$\%$ B &
\end{tabular}

${ }^{\mathrm{a}} 0$ indicates none; 1 , adequate; 2 , precise.

\section{Discussion}

Our results show that $3 \mathrm{~T}$ high-resolution imaging of the CPA allows reliable depiction of the NI. However, the prerequisite was a combination of knowledge of nervous and vascular structures with a precise definition of rating criteria and the use of modern technical equipment. The course (Fig 2), size, and characteristic intermediate position between the nervus facialis and nervus vestibularis superior (Fig 3) facilitated identification of the NI in the IAC. The change in diameter and the looped configuration or tortuosity increased the accuracy of discriminating accompanying vessels from the nerve. An additional finding (eg, the nerve shown entering of the fallopian canal) simplified decision-making (On-Line Fig 2). It was not possible to identify several roots of the NI on the same side. In addition, an approximate symmetric development was an infrequent finding (Fig 1). Therefore, a comparison of unilateral findings with the contralateral side in consideration of a potentially symmetric development of the NI did not facilitate decision-making during the evaluation process.

Previous anatomic studies reported the NI to consist of $\leq 5$ roots, ${ }^{2}$ with 1 usually predominating in size. Two roots were observed in 23\%, 3 in 16\%, 4 in $1 \%$, and 5 in $<1 \%$ of cases. A single root was observed in $59 \%$ of cases. ${ }^{2}$ This last percentage is comparable with the results of our study, with an overall identifiability of the nerve in $57.4 \%$. Because a single root is larger in diameter, we hypothesize that identification of the NI in our study may have been limited to cases consisting of 1 root or to 1 predominating root.

The following factors can impair identification of the NI: The anatomic position of the nerves in the CPA and IAC (ie, nervus facialis, nervus vestibularis superior, nervus vestibularis inferior, and nervus cochlearis) is typical in relation to each other; however, this position shows large variability ${ }^{9}$ with its clockwise (left CPA and IAC) or counterclockwise (right CPA and IAC) rotation. ${ }^{10}$ The NI cannot be fitted into this scheme because the various numbers of roots of the NI are arising from different sites between the facial nerve and the vestibulocochlear nerve. ${ }^{2}$ In approximately $20 \%$ of cases, the $\mathrm{NI}$ is adherent to the eighth nerve. ${ }^{11}$ Furthermore, fiber connections between the sensory fibers of the facial nerve (ie, the nervus intermedius) and nerve bundles of the nervus vestibularis superior and inferior within the IAC have been described. ${ }^{10}$ These can lead to a decreased acutance of the nerves. Finally, an exact knowledge of the vascularization in the CPA and $\mathrm{IAC}^{12}$ is a fundamental prerequisite for the evaluation of the NI.

The occurrence of movement, pulsation, off-resonance, or banding artifacts cannot be avoided. It was not possible to eliminate these artifacts completely from the region of interest (eg, CPA and IAC). ${ }^{13}$ According to our results of image-quality rating, artifacts did not significantly restrict the evaluation of the NI. The time of acquisition ( 8 minutes, 8 seconds) of the CISS sequence used allows implementation in clinical routine.

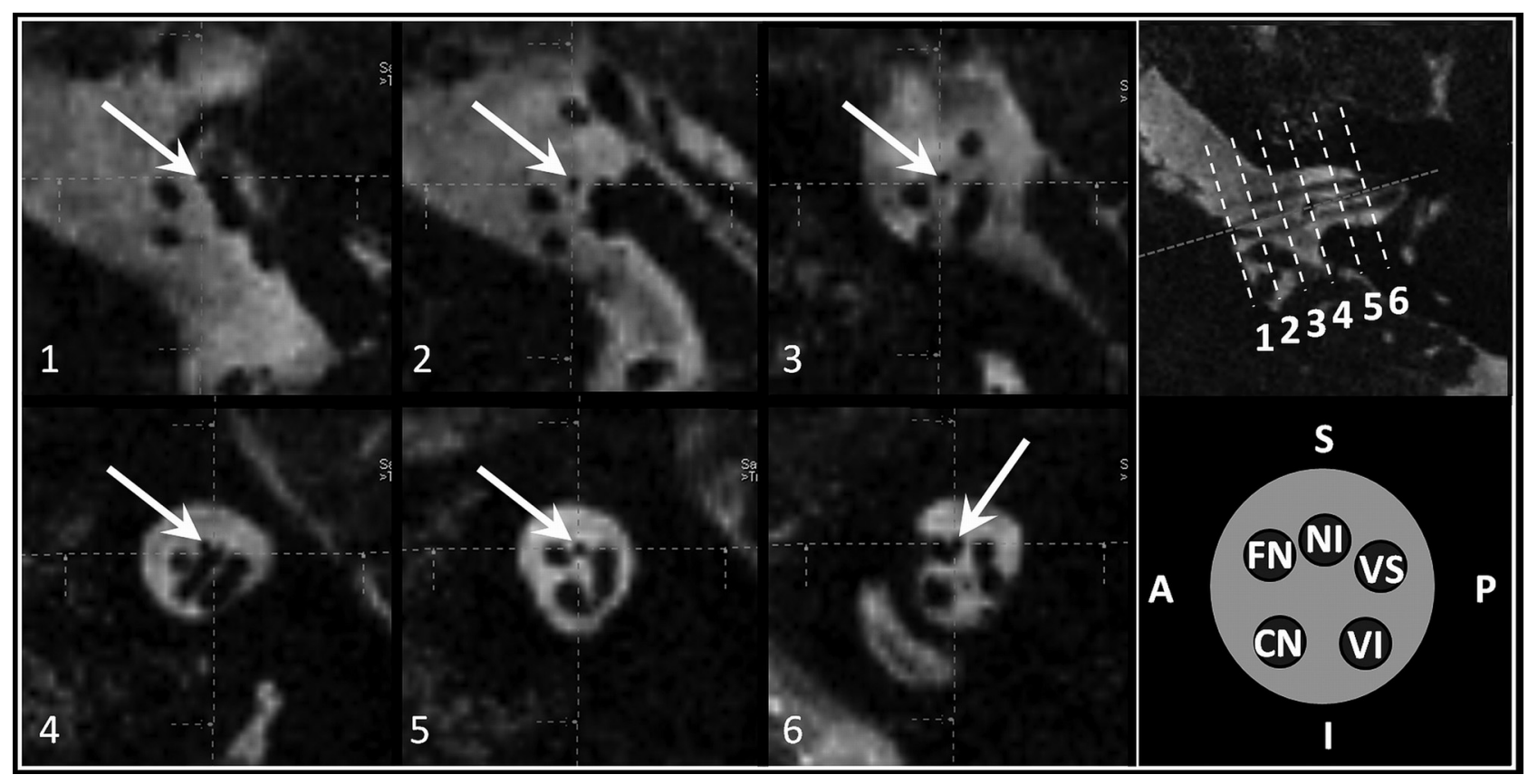

Fig 2. Parasagittal reformatted CISS sequence images (1-6) (TR, $12.18 \mathrm{~ms}$; TE, $6.09 \mathrm{~ms}$; flip angle, $50^{\circ}$ ) of the CPA and IAC at $3 T$ depicting the course of the NI and additional reference images on the right: 1) NI originates (white arrows) anterior to the nervus vestibularis superior from the brain stem, 2) NI in the middle third of the CPA between the nervus facialis (anterior) and vestibularis superior (posterior), 3) NI in the distal third of the CPA between the nervus facialis (anterior) and vestibularis superior (posterior), (4) loop of the anterior inferior cerebellar artery in the proximal IAC slightly elevating the $\mathrm{NI}$ (5) NI in the middle third of the IAC between the nervus facialis (anterior) and vestibularis superior (posterior), and 6) $\mathrm{NI}$ in the distal third of the IAC attached to the nervus facialis (anterior). Reference pictures: upper row, para-axial CISS sequence image with dotted reference lines for images; $1-6$, lower row, schematic drawing of the order of nerves (clockwise direction) in the IAC. 


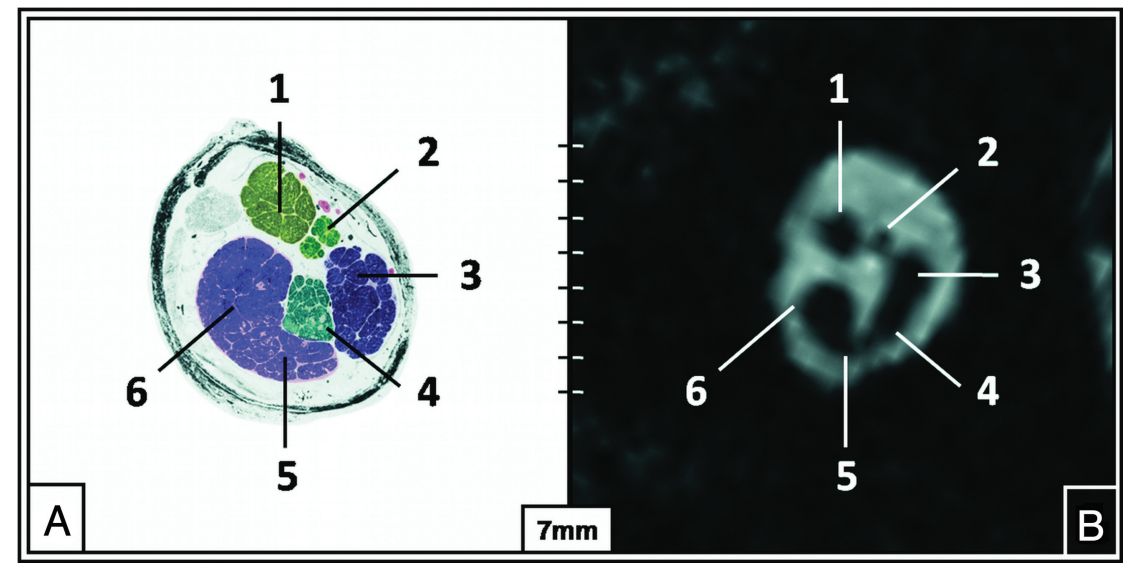

Fig 3. The intermediate position of the $\mathrm{NI}$ in the middle third of the IAC (TR, $12.18 \mathrm{~ms}$; TE, $6.09 \mathrm{~ms}$; flip angle, $50^{\circ}$ ). Semi-thin anatomic section of the left $I A C(A)$ and a correlating parasagittal in vivo CISS sequence image (B) (TR, $12.18 \mathrm{~ms} ; \mathrm{TE}, 6.09 \mathrm{~ms}$ ) at 3T depicting the nervus facialis (1), nervus intermedius (2), nervus vestibularis superior (3), nervus vestibularis inferior (4), hook of the nervus cochlearis (5), and the nervus cochlearis (6).

\section{Future Clinical Applications}

Most schwannomas of the CPA and IAC are of the nervus vestibulocochlearis. However, a study of McMenomey et $\mathrm{al}^{14}$ reported that $\leq 38 \%$ of vestibular schwannomas were mistaken for facial schwannomas, though primary facial nerve schwannomas are rare tumors with an incidence between $1.2 \%$ and $2.9 \%$ of all tumors in the CPA. ${ }^{15}$ According to our experience, most of CPA and IAC schwannomas become symptomatic (eg, peripheral facial palsy) if they reach a critical mass and are compressing and displacing adjacent nervous structures. Due to the above-mentioned aspects and to our results of asymmetric courses of the NI in most cases, it is unlikely that the technique used in this study makes it possible to differentiate midsized or large schwannomas of the facial, intermediate, or vestibulocochlear nerve. According to our results, imaging findings of the contralateral side may not be useful for surgery guidance. However, on the basis of continuous improvement of imaging procedures and due to our clinical experience in the past years, an increasing number of CPA schwannomas are diagnosed as incidental findings in an early stage of tumor development.

Modern surgical treatment modalities of schwannomas in the CPA and IAC aim to preserve both hearing and facial function. Because early tumor removal causes less damage to surrounding structures, an early and precise tumor staging is required $^{16}$ to differentiate schwannomas of the facial nerve, intermediate nerve, or vestibulocochlear nerve. Until now, reports of a CPA schwannoma found to be an NI schwannoma were limited. ${ }^{16}$ However, because of the predilection of schwannomas for sensory nerve fibers, ${ }^{17}$ it is hypothesized that the tumor origin of facial nerve schwannomas is the sensory part of the facial nerve (ie, NI). ${ }^{15}$ We believe that the technique applied in this study for identifying the NI will be a helpful tool in future clinical studies examining the verification of the latter hypothesis.

Other diseases involving the NI are hemifacial spasm because of vascular compression or schwannoma ${ }^{16}$ and NI neuralgia. ${ }^{18,19}$ The presumed etiology of NI neuralgia is analogous to that of a trigeminal tic: cross compression of the nerve at its central peripheral myelin junction. The symptoms of NI neuralgia are deep ear pain, deep face pain, or throat pain. If con- servative treatment fails, surgical treatment is a retromastoid craniectomy with microvascular decompression of cranial nerves V, IX, and X with resection of the NI. ${ }^{20}$ Therefore, imaging of the NI and of adjacent vessels may be helpful in preoperative planning.

\section{Conclusions}

With conscientious evaluation of all nervous and vascular structures in the CPA and IAC, high-resolution 3T MR imaging allows reliable depiction of the NI. Therefore in future studies, high-resolution imaging may remove uncertainties at the assignment of small CPA or IAC schwannomas to 1 of the nerves (eg, in case of vestibular-like facial nerve schwannomas). ${ }^{21}$ This may result in more frequent protection of the facial or vestibulocochlear nerve in the context of schwannoma resection, surgical treatment of NI neuralgia, or microvascular decompression. The occurrence of postoperative complications after intraoperative injury of the NI, like crocodile tears $(44 \%)$, significant reduction in the production of tears $(72 \%)$, and taste abnormality $(48 \%)$, could be reduced. ${ }^{22}$ Furthermore, a better differentiation among the facial, intermediate, and vestibulocochlear nerves may also be helpful in the assessment of other CPA lesions.

\section{References}

1. May M. Anatomy for the clinician. In: May M, Schaitkin BM, eds. The Facial Nerve: May's Second Edition. New York: Thieme; 2000:19-56

2. Oh C-S, Chung I-H, Lee K-S, et al. Morphological study on the rootlets comprising the root of the intermediate nerve. Anat Sci Int 2003;78:111-13

3. Jager L, Reiser M. CT and MR imaging of the normal and pathologic conditions of the facial nerve. Eur J Radiol 2001;40:133-46

4. Schmitt F, Grosu D, Mohr C, et al. 3 Tesla MRI: successful results with higher field strengths [in German]. Radiologe 2004;44:31-47

5. Casselman J, Mermuys K, Delanote J, et al. MRI of the cranial nerves: more than meets the eye-technical considerations and advanced anatomy. Neuroimaging Clin N Am 2008;18:197-231

6. Casselman JW, Kuhweide R, Deimling M, et al. Constructive interference in steady state: 3DFT MR imaging of the inner ear and cerebellopontine angle. AJNR Am J Neuroradiol 1993;14:47-57

7. Leblanc A. Facial nerve. In: Leblanc A, ed. Encephalo-Peripheral Nervous System: Vascularisation, Anatomy, Imaging. Berlin; Springer-Verlag; 2001:229-324

8. Thurner KH, Egg G, Spoendlin H, et al. A quantitative study of nerve fibers in the human facial nerve. Eur Arch Otorhinolaryngol 1993;250:161-67

9. Alcaraz N, King WA, Wackym PA. Endoscopy during neurotomy of the nervus 
intermedius for geniculate neuralgia. Otolaryngol Head Neck Surg 1999;121:334-35

10. Kim HS, Kim DI, Chung IH, et al. Topographical relationship of the facial and vestibulocochlear nerves in the subarachnoid space and internal auditory canal. AJNR Am J Neuroradiol 1998;19:1155-61

11. Ozdogmus O, Sezen O, Kubilay U, et al. Connections between the facial, vestibular and cochlear nerve bundles within the internal auditory canal. J Anat 2004;205:65-75

12. Yurtseven T, Savas R, Kocak A, et al. Relationship between anterior inferior cerebellar artery and facial-vestibulocochlear nerve complex: an anatomical and magnetic resonance images correlation study. Minim Invasive Neurosurg 2004;47:306-11

13. Dietrich O, Reiser MF, Schoenberg SO. Artifacts in 3-T MRI: physical background and reduction strategies. Eur J Radiol 2008;65:29-35

14. McMenomey SO, Glasscock ME, Minor LB, et al. Facial nerve neuromas presenting as acoustic tumors. Am J Otol 1994;15:307-12
15. Scheller C, Rachinger J, Prell J, et al. Schwannoma of the intermediate nerve. J Neurosurg 2008;109:144-48

16. Kudo A, Suzuki M, Kubo N, et al. Schwannoma arising from the intermediate nerve and manifesting as hemifacial spasm. J Neurosurg 1996;84:277-79

17. Cornelius JF, Sauvaget E, Huy PTB, et al. Surgical treatment of facial nerve schwannomas. Prog Neurol Surg 2008;21:119-30

18. Naganawa S, Koshikawa T, Fukatsu H, et al. Fast recovery $3 D$ fast spin-echo MR imaging of the inner ear at $3 \mathrm{~T}$. AJNR Am J Neuroradiol 2002;23:299-302

19. Bruyn GW. Nervus intermedius neuralgia (Hunt). Cephalalgia 1984;4:71-78

20. Lovely TJ, Jannetta PJ. Surgical management of geniculate neuralgia. Am JOtol 1997;18:512-17

21. Kania RE, Herman P, Tran B, et al. Vestibular-like facial nerve schwannoma. Auris Nasus Larynx 2004;31:212-19

22. Irving RM, Viani L, Hardy DG, et al. Nervus intermedius function after vestibular schwannoma removal: clinical features and pathophysiological mechanisms. Laryngoscope 1995;105:809-13 